\title{
Primary Skeletal Muscle Lymphoma Disguised as Non-Resolving Cellulitis and Deep Venous Thrombosis: A Case Report and Literature Review
}

\author{
Saad Bin Zafar Mahmood ${ }^{1 *}$, Saira Furqan ${ }^{1}$ and Muhammad Usman Shaikh ${ }^{2}$ \\ ${ }^{1}$ Department of Medicine, Aga Khan University Hospital, Pakistan \\ ${ }^{2}$ Department of Oncology, Aga Khan University Hospital, Pakistan
}

Submission: March 16, 2021; Published: March 31, 2021

*Corresponding author: Saad Bin Zafar Mahmood, Instructor - Medicine, Faculty office building, Second floor, Aga Khan University Hospital, Stadium Road, Karachi, Pakistan

\section{Abstract}

Diffuse large B-cell Lymphoma is an aggressive and prevalent form of Non-Hodgkin Lymphoma accounting for approximately around onethird of all Non-Hodgkin Lymphoma. Medical literature reports extra nodal lymphoma (ENL) to be around 20-30\% of all lymphomas mostly in central nervous system, breast, lung, intestine, liver and bone. We describe a case of a 50-year-old female diagnosed as having right arm cellulitis with DVT for last one week. With no resolution of symptoms, MRI of right shoulder and arm was done suggesting myositis with micro abscesses formation in deltoid muscle. With suspicion of malignancy a trucut biopsy of right deltoid muscle was also done along with an FDG-PET scan. The biopsy was suggestive of Diffuse Large B-cell Lymphoma. However, she didn't tolerate chemotherapy and expired after 10 days of first cycle. Primary skeletal muscle lymphomas account for approximately $0.5 \%$ of all ENL reported in literature and amazingly less than $0.1 \%$ of all lymphomas reported in extremities. Outcome of primary skeletal muscle lymphomas is very poor compared with that of lymph node lymphoma.

Keywords: Primary skeletal muscle lymphoma; DLBCL; DVT; Non-resolving cellulitis

\section{Introduction}

Non-Hodgkin's Lymphoma (NHL) is a common hematological malignancy with the greatest rate of incidence being seen in countries with a very high human development index [1]. Diffuse large B-cell Lymphoma (DLBCL) is an aggressive form of NHL and is also the most prevalent form, accounting for approximately onethird of all NHL. It is more commonly seen in the elderly population $[1,2]$. NHL is primarily a disease of lymph nodes; however, literature reports extra nodal cases to be around 20-30\% [1]. Common extra nodal sites include central nervous system, breast, lung, intestine, liver and bone. Since lymphoid tissue is absent in normal healthy skeletal muscles, extra nodal lymphoma (ENL) occurring in these muscles is quite and unusual with incidences reported to have occurred in lower extremities, pelvis and gluteal muscles $[1,3]$.

Three mechanisms have primarily been reported to be associated with skeletal muscle lymphomas; direct spread from nearby lymph nodes or bones, metastatic spread either through blood or lymphatics and least commonly being primary muscle lymphoma itself [4]. Primary skeletal muscle lymphomas are exceptionally rare accounting for only $0.5 \%$ of all ENL [3]. The

main signs and symptoms reported are swelling and pain with the presence of a mass [1]. Herein we discuss the challenges associated with the diagnosis and management of a case of primary skeletal muscle lymphoma initially presenting as non-resolving cellulitis and upper arm deep venous thrombosis (DVT) and provide a literature review associated with this disorder.

\section{Cases and Methods}

A 50-year-old hypertensive woman presented with complaints of progressively worsening right upper arm pain and swelling for the past 1 month with history of intermittent fever. She was diagnosed as having cellulitis and was on antibiotics with 


\section{Juniper Online Journal of Case Studies}

no significant relief; however, a week back she was diagnosed as having right upper arm DVT and was started on therapeutic dose of low molecular weight heparin. Her right shoulder was erythematous and exquisitely tender to touch and there was marked diffuse swelling in comparison to left arm (Figure 1).
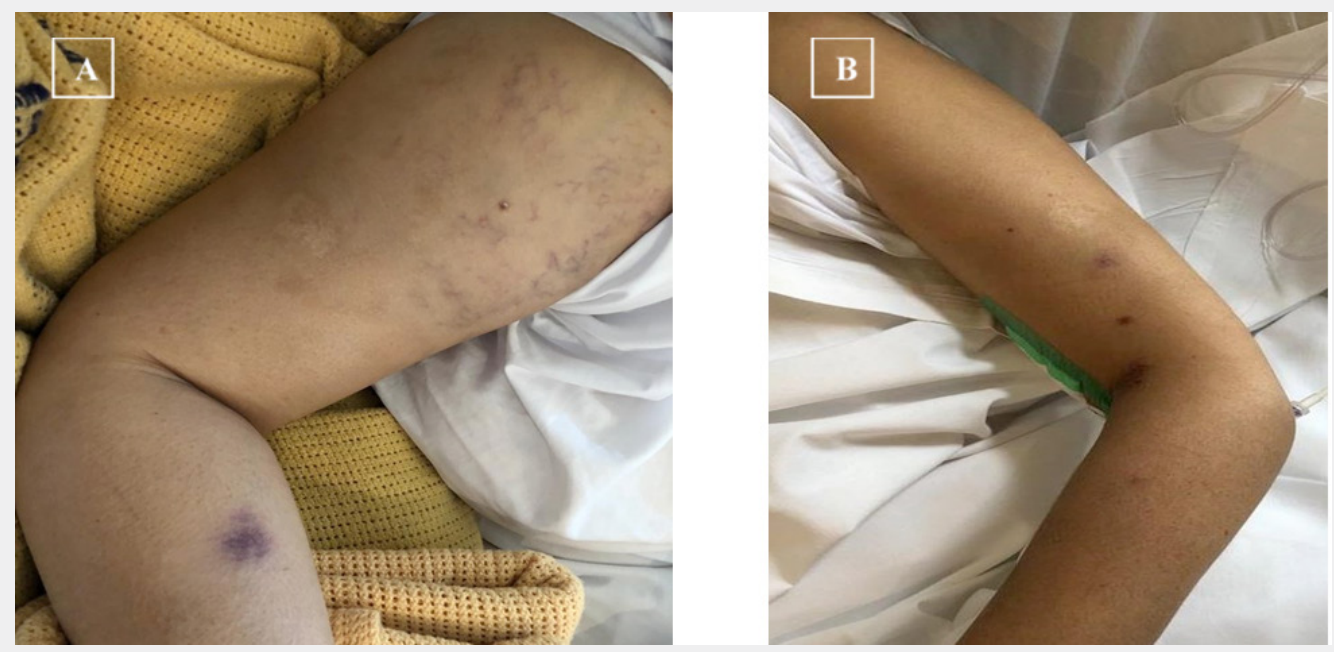

Figure 1: Comparison of right arm swelling (A) with normal left arm (B).

Blood workup showed obvious leukocytosis $\left(11500 / \mathrm{mm}^{3}\right)$ and elevated erythrocyte sedimentation rate $(115 \mathrm{~mm} / \mathrm{h}$, normal $<15 \mathrm{~mm} / \mathrm{h}$ ). Basic radiological investigation was insignificant and a Magnetic resonance imaging (MRI) of the right shoulder was done (Figure $2 \& 3$ ). Keeping in view the findings of MRI, further workup was done for brucella, tuberculosis and multiple myeloma which were all negative. Due to non-resolving nature of cellulitis and in view of underlying malignancy, positron emission tomography (PET) scan was done with fluorodeoxyglcuose (FDG) showing massive hypermetabolic soft tissue lesion involving the right shoulder and various other sites.

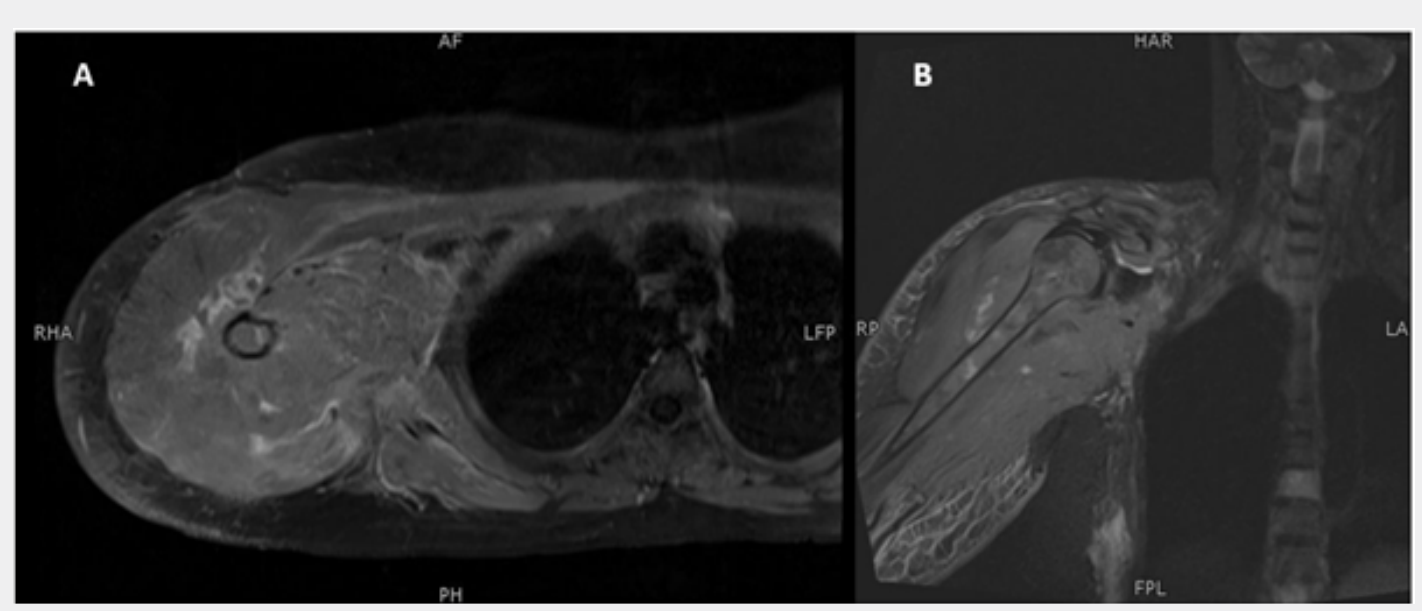

Figure 2: Axial (A) and coronal (B) MRI scan of right shoulder showed significant swelling and subcutaneous inflammatory changes in right deltoid, biceps and triceps muscle. Abnormal signal area with negative contrast enhancement was identified in the deltoid muscle suggesting myositis with microabscess formation. Multiple marrow changes in right humerus.

A trucut biopsy of right deltoid muscle was performed. Hospital stay was complicated with development of diplopia. MRI Brain was done which showed diffuse dural post contrast enhancement suggesting CNS involvement. Histopathology of trucut biopsy of right deltoid muscle was suggestive of lymphoproliferative disorder. Immunohistochemical stains performed showed positivity for LCA, CD20, PAX-5, C- myc, BCL-2, BCL-6 and CD10 with Ki-67 showing high proliferation index of near $100 \%$. Bone marrow aspiration and biopsy done for staging demonstrated normocellular marrow for age with cellular areas exhibiting diffuse infiltration with lymphoid cells and immunohistochemical staining showing diffuse positivity for CD20 (Figure 4). 


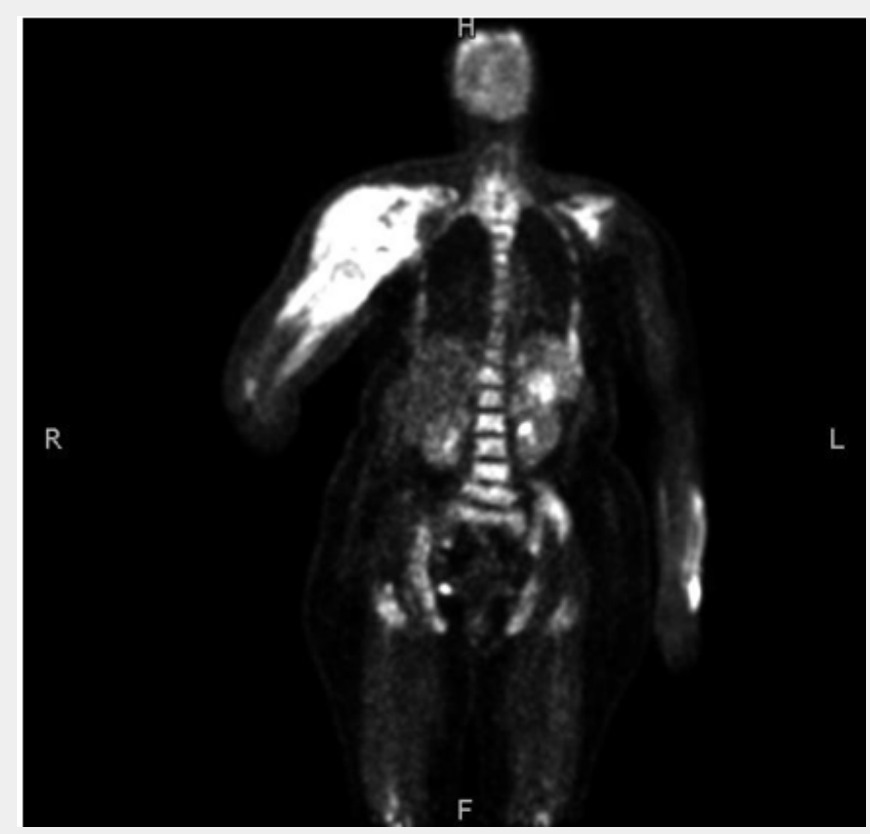

Figure 3: PET-FDG scan showing massive hypermetabolic soft tissue lesion involving the right shoulder, right scapular and proximal two third of right arm. Also showing hypermetabolic deposits in spleen, left kidney and pancreatic body along with widespread marrow deposits involving axial and appendicular skeleton.

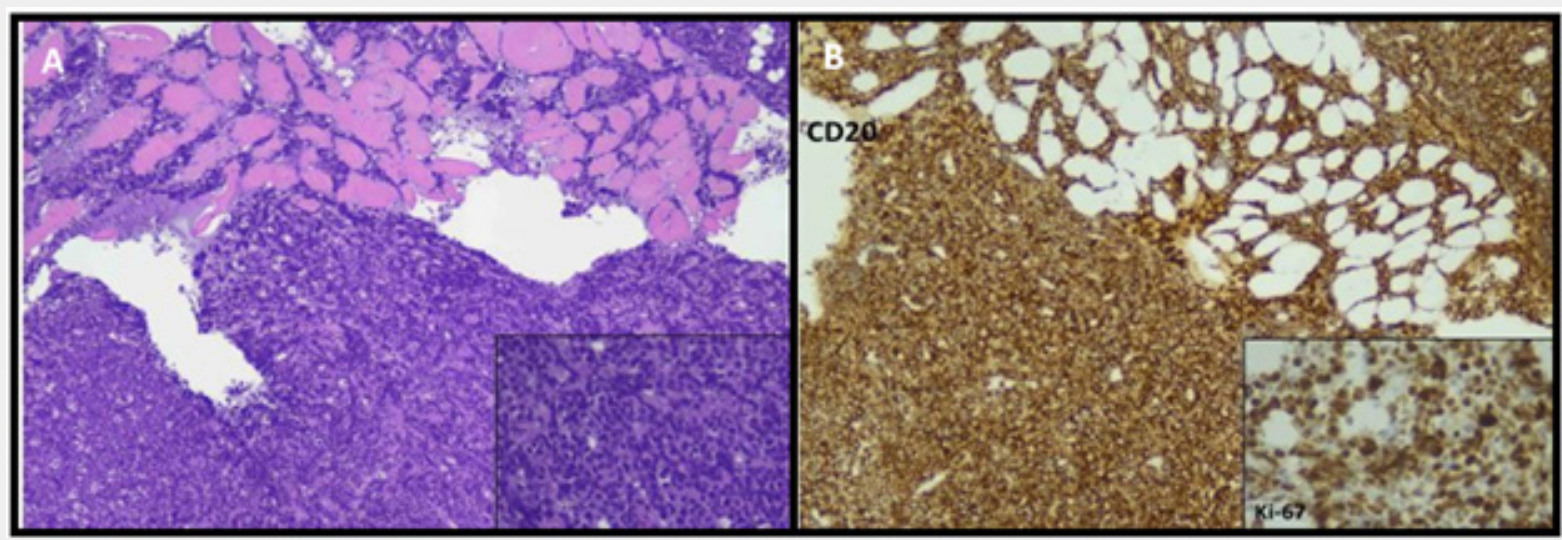

Figure 4: (A) Hematoxylin-eosin staining of sample of right deltoid muscle showing multiple cores of skeletal muscle and fibrous tissue exhibiting diffuse cellular infiltrate composed intermediate sized lymphoid cells with moderate cytoplasm; (B) Immunohistochemical staining of same sample showing positivity for CD20 with ki-67 showing high proliferation index of near $100 \%$.

Cerebrospinal fluid examination showed presence of atypical lymphoid cells. Fluorescence in- situ hybridization (FISH) was done for translocation (8:14) which was negative. A diagnosis of stage IV DLBCL was made. After completing all pre-chemotherapy requirements, patient was commenced on Rituximab, Etoposide, Prednisolone, Vincristine, Cyclophosphamide and Doxorubucin (R-EPOCH regimen) along with prophylactic antibiotics, antiviral and antifungal. However, patient completed only one cycle of R-EPOCH regimen and despite being on allopurinol, prophylactic antibiotics, antiviral and antifungal patient went into tumor lysis syndrome and severe sepsis, later developing septic shock, lactic acidosis and renal failure. Patient expired 10 days after the first chemotherapy.

\section{Discussion and Conclusion}

The entity of primary skeletal muscle lymphoma was first reported in literature by Kandel et al. [5] in 1984 [2,5]. They account for approximately $0.5 \%$ of all ENL reported in literature and amazingly less than $0.1 \%$ of all lymphomas reported in extremities [2,6]. Over a 10-year period Travis et al. [6] reported 
just 8 cases of primary muscle lymphoma among 7,000 malignant lymphomas [6]. Another case series between 1976 to 1978 studied a total of 2147 patients and found 31 primary muscle lymphomas of both B-cell and T-cell variation [4]. The hallmark presentation of primary skeletal lymphoma is swelling of muscle associated with pain and edema in the affected limb without any sign of heat and redness, along with lymphadenopathy and elevated lactate dehydrogenase $[2,7]$.

We have presented a rare case in which Skeletal muscle DLBCL localized to deltoid, biceps and triceps muscle presented initially as non-resolving cellulitis and DVT of the brachial vein without involvement of any lymph nodes. Keeping in view the rarity of the case a literature review was done for primary skeletal muscle B-cell lymphoma of the upper extremities presenting as DVT. We found only 6 cases which have reported primary skeletal muscle lymphoma of the upper extremities [3,8-12]. Of these cases 1 was localized to the forearm [8] 1 was isolated to the shoulder [9] and the remaining four were all associated with upper arm [3,10-12]. One case was reported as anaplastic large cell lymphoma [10] and the remaining five were all found to have DLBCL $[3,8,9,11,12]$. However, of all the cases only one case of primary skeletal DLBCL presented with an initial finding of DVT which was seen in an adolescent [12].

In the present study investigations for the upper arm swelling started with plain radiograph, ultrasound and then MRI as per the literature [7]. Unfortunately, in this case the findings on MRI were not very helpful. In a report of 23 skeletal muscle lymphomas MRI showed that all tumors were of intermediate T1-WI signal intensity as compared to muscle (SI), whereas $85 \%$ of tumors also exhibited intermediate T2-WI SI as compared to fat [4]. In our case PET scan was also used to identify the disease and also see the extent. PET scans have primarily been used to assess the efficacy of chemotherapy treatments. Appearance of skeletal muscle lymphomas on PET scan is non-specific and cannot be easily differentiated from sarcomas, metastases or myositis and abscesses [13]. Hence all lesions should promptly be biopsied as definitive diagnosis can only be made after biopsy which is primarily done by core biopsy but can also be performed via fine needle aspiration cytology (FNAC) [14].

Outcome of primary skeletal muscle lymphomas is very poor compared with that of lymph node lymphoma [2]. Review of literature mostly shows that the standard chemotherapy regimen of R- CHOP has been selected for skeletal muscle DLBCL. However, it does not take into account the increasing prevalent high grade B cell lymphomas (double hit and triple hit). In the present study R-EPOCH regimen was started. However, patient developed septic shock, renal failure and metabolic acidosis and expired after 10 days. Friedberg in his study also proposes that dose adjusted R-EPOCH regimen should be considered for these double hit lymphomas as it may have better outcome [15].

\section{Ethical Consideration}

Informed written consent taken from the patient's next of kin. Approval granted by the ethical review committee of the institution.

\section{Funding Source}

This research did not receive any specific grant from funding agencies in the public, commercial or not-for-profit sectors.

\section{Acknowledgement}

We would like to acknowledge the department of histopathology in making the diagnosis of primary skeletal muscle lymphoma.

\section{References}

1. John AR, Gahlot GPS, Singh BK, Jain A, Mahato A, et al (2017) Non-Hodgkin's Lymphoma with Pelvic Skeletal Muscle Involvement Presenting as Low Back Ache: An Uncommon Presentation of a Rather Common Malignancy. Indian Journal of Nuclear Medicine 32(4): 355-358.

2. Zhang L, Lin Q Zhang L, Dong L, Li Y (2015) Primary skeletal muscle diffuse large B cell lymphoma: A case report and review of the literature. Oncology letters 10(4): 2156-2160.

3. Hatem J, Bogusz AM (2016) An Unusual Case of Extranodal Diffuse Large B-Cell Lymphoma Infiltrating Skeletal Muscle: A Case Report and Review of the Literature. Case Reports in Pathology 2016: 9104839.

4. Binici DNR, Karaman A, Timur O, Tasar PNT, Sanibas AV (2018) Primary skeletal muscle lymphoma: A case report. Molecular and Clinical Oncology 8(1): 80-82.

5. Kandel RA, Bédard YC, Pritzker KP, Luk SC (1984) Lymphoma. Presenting as an intramuscular small cell malignant tumor. Cancer 53(7): 1586-1589.

6. Travis WD, Banks PM, Reiman HM (1987) Primary extranodal soft tissue lymphoma of the extremities. The American Journal of Surgical Pathology 11(5): 359-366.

7. Lee VS, Martinez S, Coleman RE (1997) Primary muscle lymphoma: clinical and imaging findings. Radiology 203(1): 237-244.

8. Leclère FM, Vogt P, Casoli V, Pelissier P, Choughri H (2015) Double crush syndrome of the median nerve revealing a primary non-Hodgkin's lymphoma of the flexor digitorum superficialis muscle. Chir Main 34(5): 256-259.

9. Marotta D, Sgambato A, Cerciello S, Magarelli N, Martini M, et al. (2008) Soft tissue non-Hodgkin lymphoma of shoulder in a HIV patient: a report of a case and review of the literature. World Journal of Surgical Oncology 6: 111.

10. Ishii E, Honda K, Nakagawa A, Urago K, Oshima K (2000) Primary CD30/Ki-1 positive anaplastic large cell lymphoma of skeletal muscle with $\operatorname{der}(17) \mathrm{t}(1 ; 17)(\mathrm{q} 11 ; \mathrm{p} 11)$. Cancer genetics and cytogenetics 122(2): 116-120.

11. Ueno A, Kuribayashi K, Iyama S, Matsunaga T, Kikuchi S, et al. (2005) Two cases of primary skeletal muscle lymphoma, and a review of the literature. [Rinsho ketsueki] The Japanese journal of clinical hematology 46(10): 1141-1145.

12. Dhillon RK, Spahr CD (2010) Two cases of upper-extremity swelling: Paget-Schroetter syndrome and non-Hodgkin lymphoma. Pediatric Emergency Care 26(4): 290-292. 
13. Hwang S (2008) Imaging of lymphoma of the musculoskeletal system Radiologic clinics of North America 46(2): 379-396.

14. Bozzola C, Boldorini R, Ramponi A, Monga G (2005) Fine needle aspiration cytology in the diagnosis of non-Hodgkin's lymphomas of the muscle: a report of 2 cases. Acta cytologica 49(2): 213-218.
15. Friedberg JW (2017) How I treat double-hit lymphoma. Blood 130(5): 590-596.

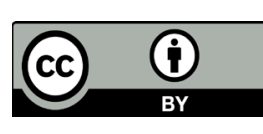

This work is licensed under Creative Commons Attribution 4.0 License DOI: 10.19080/JOJCS.2021.12.555830

\section{Your next submission with Juniper Publishers will reach you the below assets}

- Quality Editorial service

- Swift Peer Review

- Reprints availability

- E-prints Service

- Manuscript Podcast for convenient understanding

- Global attainment for your research

- Manuscript accessibility in different formats

( Pdf, E-pub, Full Text, Audio)

- Unceasing customer service

Track the below URL for one-step submission

https://juniperpublishers.com/online-submission.php 